\title{
Preparing Engineering Students to Work on Taboo Topics in the Service of Communities
}

\section{Ms. Devika Patel, Stanford University}

Devika is currently fourth year undergraduate at Stanford University studying Mechanical Engineering: Product Design with honors in Education and Public Service. She will be continuing her studies at Stanford through an M.S. in Community Health and Prevention Research. She is an undergraduate researcher at the Kometsky Global Collaboratory, where she is looking at working with taboos in hygiene and sanitation in engineering contexts. 


\section{Preparing Engineering Students to Work on Taboo Topics in the Service of Communities}

\section{Introduction}

According to the WHO, 2.4 billion people lack access to proper sanitation resources. ${ }^{1}$ Faced with a growing problem, engineers, locally and internationally, have responded to this crisis through avenues such as the Gates Reinvent the Toilet Challenge. In the case of the Toilet Challenge, engineers were able to create what were seen as "practical" toilets that convert waste into energy. However, each toilet cost upwards of $\$ 1000$ and required infrastructure and technology not available in their target communities, thus presenting a huge drawback in fighting the sanitation stigma. $^{2}$ There is much to learn from instances like the Toilet Challenge. To put trained and qualified engineers out into the world, it is necessary to supplement engineering education with culturally aware project-based curriculum. In ensuring global impact, meshing together the technical, social and cultural aspects of an engineer's humanitarian effort is crucial.

However, this is not frequently seen in the context of engineering projects with the international community. The need for these specific skills and research is even more integral in the study of stigmatized, or taboo, topics that engineers may find themselves addressing in their projects, where the approach can be key to the success of an intervention. Taboo issues consist of acts that are considered to be forbidden, unmentionable, unthinkable in certain societal settings. ${ }^{3}$ Taboo subjects around the world consist of anything from HIV/AIDS, issues surrounding abuse, etc. The taboo subject of focus for this paper is that of menstruation and menstrual hygiene, a taboo subject directly a part of the sanitation crisis in India. When having to interact with taboo subjects, as they vary greatly from culture to culture, engineering students must be prepared and competent in their interactions surrounding their community partners and themselves.

\section{Research Questions}

While people from around the world have worked with and created technologies around the menstrual hygiene space, little is understood of the role international engineers, and in particular, the role engineering students could play in the research and design behind such products. This paper poses the following research question: What factors should engineering students be prepared to handle and address in learning how to apply their skills involving taboo topics, such as menstrual hygiene, when working with communities outside of their own? Notably, progress is being made through the participation of major foundations and nonprofits encouraging members to consider "engineering for social good" along with the prevalence and reputation of university and professional based chapters of Engineers without Borders or Engineers for a Sustainable World. ${ }^{4}$ However, these advances have not permeated through revitalizing traditional engineering education practices.

\section{Cultural Competence, Engineering and Taboo}

To fill this gap in engineering education of culturally and contextually relevant research, there is a need for a new social justice literacy in engineering. ${ }^{5}$ Yet, this is not the case when looking at the professionalization of the engineering career: "In this point, the professional association recognises the need for engineers to play a broader social and environmental role. Yet the emphasis on technical competencies sends a powerful message to engineering educators, highlighting the primacy of technical knowledge in a crowded curriculum". 6 The need for this 
new literacy to highlight how one might gauge a sense of social responsibility has been formalized through two metrics. The metrics of assessment are twofold: first, global preparedness, or "the readiness to engage and effectively operate under uncertainty in different cultural contexts to address engineering problems" and second, global competency, or "development of one's skills and attitudes in successfully interacting with persons of diverse backgrounds" have recently been brought forward into the academic circles in engineering education research. $^{7}$

The notions of global competence and global preparedness are especially relevant and critical when working with what are considered taboo issues. This paper will explore an engineering student's experience in working with a rural community, that will inform requirements for "global competence and preparedness" in working with menstrual hygiene taboo. It also provides the community with the experience in working with external, international professional engineers in an equitable and reciprocal relationship.

\section{Case Study of Menstrual Hygiene in India}

One such taboo subject around the world is that of menstruation and menstrual hygiene. This process is necessary in the healthy functioning of a woman's reproductive system, but has been, for various reasons, cast as an undesirable and unmentionable occurrence in the lives of women worldwide. As a case in point, in India, menstruation restricts women from certain activities. Thought of as dirty and impure, menstruation restricts women from doing their jobs and living a normal life under the control of the taboo. The impact of these taboos on women in India are grave. Studies have also revealed that girls stated they performed poorly in school if they attended during menstruation, due to the worry that their male peers would find out. ${ }^{8}$ Additionally, studies have shown that use of unhygienic sanitary products during menses is linked to higher rates of urinary and reproductive tract infections. ${ }^{8}$ All in all, the current state of menstrual hygiene in India is concerning at least.

Efforts have been undertaken in exploring innovative approaches to combat the issues surrounding menstrual hygiene. Other than lack of knowledge of menstrual hygiene management and practices, another key point of underdevelopment is access to menstrual hygiene resources. It is in these interventions where engineering and design become more apparent in their traditional sense. One famous example of this is that of Arunachalam Muruganantham, a social entrepreneur from South India, who invented a machine that can manufacture low cost sanitary napkins. ${ }^{9}$ His work has inspired many others around India. For instance, a textile engineer created a low-cost incinerator for the safe disposal of used sanitary pads. ${ }^{10}$ These are just two examples of social enterprises that are emerging all over India regarding the manufacturing and disposal of used sanitary pads and napkins. Engineers play a crucial role in the design and manufacturing aspects of these products.

\section{An Autoethnography on Working with Taboos}

While people from around the world have worked with and created technologies around the menstrual hygiene space, little is understood of the role of international engineers, and in particular, engineering students, in the research and design behind such products. In looking at these gaps in academic literature, this paper will employ the use of autoethnography, the process of using one's own experience and narrative to formulate and piece together the context of their experience in a reflective and analytical manner. ${ }^{11,12}$. This methodology will prove important in 
understanding the context of the taboo issue the engineering student will confront. To assess preparedness for working with taboo subjects, the metrics of global preparedness and global competency will be examined and tested through the use of an autoethnographic study designed to examine the experience. These measures will be used by the author, and the data gathered from interviews from local stakeholders in a rural village in India. The author's autoethnography will be used to capture a rich set of experiences and will serve to be an example and backdrop with which to build a framework in understanding taboo issues and preparing engineering students to be cognizant and ready to face such issues appropriately.

\section{Method}

The author is working with a community through the assistance of the Environmental Sanitation Institute, a non-governmental organization based near Ahmedabad in western India. Focused on bringing safe hygiene and sanitation practices to the rural poor, the NGO uses a variety of methods to work with the local village communities to teach and spread best practices - such as the use of a toilet, which includes designing and building them in the village, personal hygiene practices and working with the women and health workers to train them on common illness prevention and care. The author spent a total of four weeks in the field, as well as one and half year communicating with the NGO through Skype. The first field visit was done in August of 2014, with the second field visit done in December 2015. Through the field visits, the author worked with the community organization to locate key villages and stakeholders to begin learning from the women in the villages regarding the taboo surrounding menstruation.

During the second field visit, the author interviewed ten women from a small village near Ahmedabad, which right outside the NGO headquarters. This village had recently opened a community center, and work from the NGO has been ongoing for almost two years. Almost every household in this village has a toilet and access to clean water. The author spent their time in the village conducting semi-structured interviews, mostly with women and some community leaders. The interviews were conducted in Gujarati, recorded to be translated and transcribed at a later date. It is important to note that the author has native language fluency in the language spoken, which in this case, is Gujarati. The interview questions were targeted towards understanding the nature of the menstrual hygiene taboo as they see it and then exploring ways the community members would find it most useful and effective for engineering students to get involved in providing solutions to issues in this problem space. The data that was collected in December 2015 is undergoing a thorough analysis. From journal entries and personal field notes, the author will be understanding their own experience and extracting any trends of improvement, or lack thereof, in their methods of working with the community. This will be measured against the metrics of global preparedness and global competency. Data will then be analyzed by coding for looking for various themes, adapted from the work of Parkinson et al., (2009). ${ }^{13}$

The second part of the data analysis comes from the interviews themselves. These interviews will be coded for the major themes they either share or don't share as design requirements and criteria for effective engineering projects with the adapted dimensions from Parkinson et al. ${ }^{13}$ By analyzing this data, key trends will emerge and provide useful insights in developing a framework of factors engineering students need to be prepared to work with and handle in dealing with taboo topics in the service of communities.

\section{Preliminary Results}


Preliminary results from the author's autoethnography point to the necessity of being adaptive and receptive to feedback while in the field. The author is fluent in the local language of the community, which eased potential communication barriers, and is female, lowering inhibitions regarding the gender sensitivity surrounding menstrual hygiene. However, in speaking about a taboo subject, coming in as an outsider, being cognizant of the author's position with respect to the community and responding appropriately became critical because of the author coming from Stanford University and the privilege that is associated with that. In the case of this rural village, it was important that the author spent time outside of the interviewing process learning from the local village people, participating in their daily activities, such as chores, playing with children, and eating meals with community members. While interviewing, working with the community organization in finding willing participants and running potential questions by their staff was necessary in understanding that some of the pre-scripted questions were not well worded. For instance, in order to level the exchange of information, the author found that the tension that sometimes arose from the conversation came from the participant feeling as if they were getting nothing in return for talking about something forbidden. In response, the author offered to share personal information and stories of their own experiences with menstruation, making the interview more of a conversation. This diffused the nature of the taboo conversation, and allowed the author and participant to feel more comfortable in talking about how engineering students may assist their communities in the space of menstrual hygiene. This key insight, including the validation of other guidelines set by the metrics of global preparedness and global competency, such as language competence, are just two instances from which best practices for future engineering students can be discerned. As the best practices are written by the author, a current engineering student, the guidelines will be useful in making a more relatable, transparent, and meaningful framework for engineering students working in similar situations.

\section{Preliminary Conclusions}

Engineering education, or the formal training of engineering students, is integral in preparing these engineers to go out into an increasingly globalized world. From this, comes a need to bridge the gap between engineering education theory and practice, particularly, the study of whether these types of interactions, between engineering students and international communities, are worthwhile and equitable for both parties. Traditional engineering science programs focus on technical knowledge. Though this is a very important aspect, there is an increasing need to make space for the addition of nontechnical skills: according to Engineers without Borders Executive Director, Cathy Leslie, "The world has become much more global, given the Internet and access and so I think it is not so much that the need has changed, it is that the opportunities in the market have changed...And our educational structures are saying, 'We need to better prepare for this" ". ${ }^{14}$ Following this notion of preparation of engineering educational structures, the concept of cultural sensitivity is usually mentioned, yet its true meaning and significance are not well understood. To remedy this, extensive research into the context in which the product is being implemented - research that examines contextual factors, local stakeholders and cultural sensitivity, to name a few, needs to be conducted. Using autoethnography, semi-structured interviews, and personal journal entries, this research hopes to create a preliminary framework for engineering students interested in working on taboo subjects in communities other their own and contribute to expanding engineering education on this front.

\section{Acknowledgements}


First and foremost, I would like to thank Dr. Bhavna Hariharan, my professor and advisor, for her crucial guidance over the past two years throughout the research and writing processes. In addition, I would like to thank Dr. John Willinsky, Dr. Christine Wotipka, and Kamran Naim for their guidance through the research process and continuously challenging my assumptions. Last, but certainly not least, I would like to thank my collaborators and the village members in India who made this research possible, for their open arms, open hearts, and open minds.

\section{References}

1. Bulletin of the World Health Organization 2014, 92, 470-471.

2. Kass, J. (2013, November 18). Bill Gates Can’t Build a Toilet. New York Times. Retrieved December 10, 2015.

3. Sorcar, P. (2009). Teaching Taboo Topics Without Talking About Them: An Epistemic Study of a New Approach to HIV/AIDS Prevention Education in India (Doctoral dissertation). Retrieved from TeachAIDS.

4. Hariharan, B. (2011). Innovating Capability for (Deweyan) Continuity of Inquiry in the Face of (Zimbardoean) Discontinuity Within the Context of Engineering Education Research: Fostering Collaborations with Underserved Communities in the Developing Regions of the World. Department of Mechanical Engineering Stanford University.

5. Riley, D. (2008). Chapter 4. Toward a More Socially Just Engineering. In Engineering and social justice (p. 111). San Rafael, Calif.: Morgan \& Claypool.

6. Cumming-Potvin, W., Currie, J., (2013), Towards New Literacies and Social Justice for Engineering Education, International Journal of Engineering, Social Justice, and Peace, 2(1), 21-37.

7. Streiner, S., Cunningham, S., Huang, S., Levonisova, S., Matherly, C., Besterfield-Sacre, M., . . Kotys-Schwartz, D. (2014). Exploring Engineering Education in Broader Context: A Framework of Engineering Global

Preparedness. American Society of Engineering Education.

8. Mahon, T., \& Fernandes, M. (2010). Menstrual hygiene in South Asia: A neglected issue for WASH (water, sanitation and hygiene) programmes. Gender \& Development, 99-113.

9. Venugopal, V., \& Abhi, S. (2013). A New White Revolution: Case Study of a Social Entrepreneur.

10. Joshi, S. (2015, January 6). How Swati Made Menstruation Not Only More Hygienic But Also More Profitable For Rural Women, The Better India. Retrieved December 10, 2015.

11. Spry, T. (2001). Performing Autoethnography: An Embodied Methodological Praxis. Qualitative Inquiry,706732.

12. Ellis, C. (2004). The Ethnographic I: A Methodological Novel about Autoethnography.

13. Parkinson, A. (2009). The Rationale for Developing Global Competence. Online Journal for Engineering Education, 4(2).

14. Lieberman, Amy. (2015). "5 Fast Tips for Your Career as a Global Engineer." Devex. 